\title{
El Burnout Académico Percibido en Universitarios; Comparaciones por Género
}

Judith M. Rodríguez-Villalobos, Elia V. Benavides, Martha Ornelas y Perla J. Jurado*

Universidad Autónoma de Chihuahua. Facultad de Ciencias de la Cultura Física. DES Salud CAs 101 y 121. Calle Escorza 900, CP 31000 Chihuahua, Chih. México. (e-mail: jurodrig@uach.mx, ebenavides@uach.mx, mornelas@uach.mxy pjurado@uach.mx)

* Autor a quien debe ser dirigida la correspondencia.

Recibido Dic. 13, 2018; Aceptado Feb. 25, 2019; Versión final Abr. 1, 2019, Publicado Oct. 2019

\begin{abstract}
Resumen
El objetivo de esta investigación consistió en comparar los perfiles de burnout percibido de mujeres y hombres universitarios en la Universidad Autónoma de Chihuahua, México. La muestra total fue de 1180 participantes; 598 mujeres y 582 hombres, con una edad media de 20.50 años y 20.84 años, respectivamente. El abordaje adoptado en la investigación se enmarcó dentro de un enfoque cuantitativo con un diseño descriptivo tipo encuesta. Todos los participantes contestaron el Inventario de Burnout para Estudiantes de Maslach. Los resultados del análisis multivariante de varianza, seguido por los análisis de varianza univariados, muestran que las mujeres en comparación con los hombres reportan mayores niveles de burnout académico en las tres dimensiones estudiadas (Agotamiento, Cinismo y Eficacia). Las diferencias encontradas entre hombres y mujeres con respecto a su percepción de burnout académico, sugieren que al diseñar cualquier tipo de intervención que tenga como objetivo la disminución del burnout percibido habrá que tomar en cuenta a la variable género.
\end{abstract}

\section{Academic Burnout Perceived in Mexican University Students Comparisons by Gender}

\begin{abstract}
The aim of the present study was to compare the profiles of academic burnout between men and women students of the University Autónoma de Chihuahua, México. A total sample of 1180 participants, 598 women and 582 men, with an average age of 20.50 years and 20.84 years, respectively. A quantitative approach with a descriptive and transversal survey design was used. All the participants completed the Maslach Burnout Inventory Student Survey. The results of the one-way multivariate analysis of variance, followed by the oneway univariate analysis of variance, showed that compared with men, women report higher levels of academic burnout in the three dimensions studied (Exhaustion, Cynicism and Efficacy). The differences found between men and women with respect to their perception of academic burnout, suggest that when designing any type of intervention that has as objective the reduction of perceived burnout, the gender variable should be taken into account.
\end{abstract}

Keywords: burnout; student's beliefs; gender differences; self-perception 


\section{INTRODUCCIÓN}

El término burnout se utiliza como una metáfora para explicar que la energía de las personas se drena, hace referencia también a la sofocación en un incendio o cuando una vela se va extinguiendo y su brillo se va apagando; donde con el tiempo las personas que experimentan burnout pierden la capacidad de proporcionar contribuciones que tengan impacto en su entorno (Shaufeli et al., 2009). La primera vez que se hizo referencia al concepto de burnout fue en 1960 en una novela de Graham Greene titulada "A Burnout case", pero es el psicoanalista Freudenberger (1974) quien relaciona esta expresión al estado de fatiga y frustración obtenido de la dedicación a una causa, estilo de vida o relación que no contribuye en el refuerzo esperado por el sujeto que lo desarrolla; Freudenberger define el burnout como una combinación de cansancio emocional crónico, fatiga física, pérdida de interés por la actividad laboral, baja realización personal y deshumanización en el cuidado y atención. Este fenómeno que se retrató en los primeros artículos no había sido del todo desconocido, pero rara vez se reconoció o incluso se discutió abiertamente. En algunas ocupaciones era casi un tema tabú porque se consideraba que equivalía a admitir que en ocasiones los profesionales pueden (y lo hacen) actuar "sin profesionalidad". La reacción de muchas personas fue negar que tal fenómeno existiera o, si existiera, atribuirlo a una minoría muy pequeña (pero claramente perturbada mentalmente). Esta respuesta hizo difícil, al principio, tomar en serio cualquier trabajo sobre el agotamiento. Sin embargo, después de que se publicaron los artículos iniciales, hubo un cambio importante en la opinión. Los profesionales de los servicios humanos dieron un apoyo sustancial tanto a la validez del fenómeno como a su importancia como riesgo laboral. Una vez que el agotamiento fue reconocido como un problema legítimo, comenzó a atraer la atención de varios investigadores (Maslach, 2017).

De hecho, el concepto de burnout se ha ampliado a otros ámbitos, pues se ha encontrado que se puede presentar en cualquier circunstancia, es decir, que trasciende a otros contextos (Gorski, 2018; Richards et al., 2018). Como es sabido los estudiantes universitarios se encuentran expuestos a muchas demandas presentadas por su contexto educativo, el aprendizaje, el rendimiento académico, la sobrecarga de trabajo, la presión con el tiempo, la falta de oportunidades para la autogestión, la evaluación frecuente, la competencia entre compañeros, la irrelevancia percibida de contenidos, una enseñanza deficiente, las malas relaciones, etc.; todas estas pueden constituirse como causas de estrés el cual puede llegar a niveles crónicos; es por ello que se considera que el estudiante en el ámbito académico es uno de los más vulnerables a la aparición del burnout académico (Karimi et al., 2014; Moneta, 2011; Vizoso y Arias, 2016). Así mismo se ha demostrado en diversos estudios que los estudiantes sufren de burnout académico (Casanova et al., 2016; Hinrichs et al., 2016; Kristanto et al., 2016; Morgan et al., 2014; Seibert et al., 2017); y que además el burnout les afecta en el rendimiento académico (Fiorilli, et al., 2017; Jahedizadeh et al., 2016). Asimismo, los estudiantes que sufren el síndrome de burnout muestran más altos niveles de ausentismo, poca motivación hacia sus clases, menor compromiso escolar y presentan síntomas depresivos (Fiorilli et al., 2017); también este síndrome produce una mala regulación de las emociones (Seibert et al., 2017). Así pues, el estrés en la universidad se ha relacionado con afectar negativamente la vida del estudiante en una variedad de formas que causan el síndrome de burnout, es un problema prevalente que afecta a los estudiantes dentro de la educación superior; el síndrome de Burnout pues, puede hacer que los estudiantes encuentren estrés en el curso, soledad, emociones negativas de aprendizaje, entre otros (Castellanos, 2018).

Por otro lado, el desarrollo teórico del burnout recibe un fuerte impulso conceptual en la década de los 80 , se divide en dos diferentes enfoques que predominan en el campo de estudio de este síndrome (Morgan et al., 2014); el enfoque tridimensional caracterizado por agotamiento emocional, despersonalización (cinismo), y realización personal; y el enfoque unidimensional con la dimensión de agotamiento emocional (Pérez-Mármol y Brown, 2018). Sin embargo, los autores Gil-Monte et al. (2009) proponen un modelo de cuatro factores para caracterizar el burnout: el primero es ilusión por el trabajo que se refiere al deseo del individuo de alcanzar las metas laborales porque supone una fuente de placer personal; el segundo es desgaste psíquico definido como la aparición de agotamiento emocional y físico debido a que en el trabajo se tiene que tratar a diario con personas que presentan o causan problemas; tercero, la indolencia que se refiere a la aparición de actitudes negativas de indiferencia y cinismo hacia los clientes de la organización; y el cuarto factor es culpa definido como la aparición de sentimientos de culpa por el comportamiento y las actitudes negativas desarrolladas en el trabajo, en especial hacia las personas con las que se establecen relaciones laborales.

Por su parte los investigadores Mészaros et al. (2013) probaron un modelo bifactorial que incluye un factor global de agotamiento y tres factores específicos: agotamiento emocional, despersonalización y baja realización personal; donde mostraron que una proporción relativamente grande de la variación común en la puntuación total de agotamiento se explica por el factor agotamiento global, lo que sugiere que debería asignarse mayor importancia a una única dimensión de agotamiento. En cuanto a los estudios realizados en burnout académico el enfoque tridimensional es el más predominante. En este enfoque el agotamiento emocional se refiere al sentimiento de haber agotado los recursos emocionales de uno, y es considerado como el componente de estrés individual básico del burnout. La despersonalización se refiere a las respuestas 
negativas, cínicas o excesivamente desinteresadas hacia otras personas en el trabajo, éste es el componente interpersonal del síndrome. Y finalmente la baja realización personal que alude a sentimientos de disminución de la competencia de uno mismo, así como la productividad, y representa el componente de autoevaluación del burnout (Moneta, 2011). Referente a la medición del burnout se han empleado diferentes técnicas llegando a ser la más común el empleo de cuestionarios y autoreportes, de todos los instrumentos generados el Inventario de Burnout de Maslach en sus diferentes versiones y adaptaciones ha sido el estándar de oro para este propósito; este inventario mide el burnout a través de tres dimensiones: agotamiento, cinismo y eficacia académica, la primera dimensión mide la fatiga sin hacer referencia a los sentimientos causados por otras personas; la segunda dimensión refleja la actitud personal pero distante en relación a los estudios; y la tercera dimensión hace referencia a qué tan eficaz se siente la persona en relación a sus estudios para resolver problemas, hacer contribuciones, alcanzar sus metas, etc.

El burnout puede afectar de diferente manera de acuerdo al género (Lebares et al., 2018; Tement et al., 2016); varios autores han encontrado que las mujeres son las más afectadas por el síndrome de burnout, por ejemplo Acosta et al. (2016) realizaron un estudio transversal y descriptivo para conocer la presencia del burnout en 230 estudiantes de medicina empleando la Escala Unidimensional del Burnout Estudiantil (EUBE), encontrando su predominio en el sexo femenino; así mismo, Go et al. (2018) en su investigación con 393 estudiantes de medicina koreanos, quienes utilizaron la escala de Maslach Burnout Inventory reportaron que las mujeres tuvieron puntuaciones significativamente más altas que los hombres; también Serrano et al. (2016) encontraron resultados similares en una muestra de 180 estudiantes de seis facultades de medicina colombianas empleando el test Maslach Burnout Inventory-Student Survey (MBI-SS); algunos concuerdan en afirmar que en la dimensión de agotamiento emocional es donde puntúa más alto el género femenino (Herrera et al., 2016).

En cambio, Portolés y González (2015) reportan un agotamiento emocional y despersonalización más alto en los hombres, mientras que las mujeres presentan una eficacia académica más alta en comparación con los hombres. En relación a la dimensión de cinismo los hombres presentan puntuaciones significativamente más altas (Portolés y Hernández, 2016). En tanto que Lebares et al. (2018) afirman que son los varones en quienes la despersonalización es mayor que en las mujeres. Por el contrario, otros autores afirman que no existe diferencia significativa entre géneros (Peralta-Ayala y Moya, 2017). Sin embargo, dichas diferencias de burnout entre hombres y mujeres también se pueden deber a la cultura, la profesión, el trabajo, otras tensiones encontradas y la medida utilizada para evaluar (Malach-Pines y Ronen, 2016). La clave para el manejo del burnout es la identificación temprana y la intervención rápida y efectiva (Aulak et al., 2016); ya que es un síndrome silencioso y nocivo (Acosta et al., 2016); por tanto, el objetivo de este estudio es analizar las diferencias por género a través de los factores que componen el Maslach Burnout Inventory Student Survey (MBI-SS) adaptado por Shaufeli et al. (2002).

\section{METODOLOGÍA}

Se describen los participantes, se dan detalles del instrumento utilizado y se explica el procedimiento de recolección de datos, para luego detallar cómo se realiza el análisis de datos.

\section{Participantes}

La muestra de 1180 participantes, 598 (50.7\%) mujeres y $586(49.3 \%)$ hombres, todos alumnos y alumnas de la Facultad de Ciencias de la Cultura Física de la Universidad Autónoma de Chihuahua. Las edades de las mujeres fluctúan entre los 18 y 28 años, con una media de 20.50 y una desviación estándar de 1.83 años; y las de los hombres fluctúan también entre los 18 y 28 años, pero con una media de 20.84 y una desviación estándar de 1.98 años. La muestra se obtuvo mediante un muestreo por conveniencia, tratando de abarcar la representatividad de los diferentes semestres de las licenciaturas que se ofrecen en la la Facultad de Ciencias de la Cultura Física de la Universidad Autónoma de Chihuahua.

\section{Instrumento}

Inventario de burnout para estudiantes de Maslach (Maslach Burnout Inventory Student Survey: MBI-SS) adaptado por Shaufeli et al. (2002), este instrumento se tradujo al español con la ayuda de expertos y se volvió a traducir a su idioma original inglés para corroborar su adecuada traducción. El inventario de burnout para estudiantes de Maslach consta de 15 ítems agrupados en tres subescalas: agotamiento con cinco ítems, el cual se mide por elementos que se refieren a la fatiga pero no hacen referencia a otras personas como la fuente de esos sentimientos (me siento emocionalmente agotado por mis estudios, me siento usado al final de un día en la universidad, me siento cansado cuando despierto en la mañana y tengo que enfrentar otro día en la universidad, estudiar o asistir a una clase es realmente estresante para mí, me siento quemado por mis estudios); cinismo con 4 ítems, refleja la actitud distante hacia los estudios, no necesariamente con otras 
personas (me he vuelto menos interesado en mis estudios desde que empecé la universidad, me he vuelto menos entusiasmado respecto a mis estudios, me he vuelto más cínico sobre lo que mis estudios pueden ser, dudo sobre la importancia de mis estudios); y eficacia académica con 6 ítems (por ejemplo: puedo resolver eficazmente los problemas que surgen en mis estudios, creo que puedo hacer una contribución efectiva en las clases que asisto, en mi opinión soy un buen estudiante, me siento estimulado cuando alcanzo mis metas estudiantiles, he aprendido muchas cosas interesantes a lo largo de mis estudios, durante la clase me siento confiado de que soy efectivo en hacer las cosas) donde puntuaciones altas en agotamiento y cinismo, y bajas en eficacia profesional son indicativas de mayor burnout. En la consistencia interna reportada de la muestra tanto para España, como en Portugal y los países bajos el alpha de Cronbach fue mayor de .74 en agotamiento, en cinismo y en eficacia académica, excepto en los países bajos en el factor eficacia académica con un alpha de Cronbach de .67.

Para nuestro estudio se hicieron dos adaptaciones a la versión de Shaufeli et al. (2002): i) Primera adaptación, en la versión Shaufeli y colaboradores se puntúa con siete opciones de respuesta de (0) nunca a (6) siempre; en la versión utilizada en la presente investigación el sujeto elige entre 11 posibles respuestas. Conjugamos la escala original con nuestra versión para que quedara de la manera siguiente: nunca (0), casi nunca (1, 2 y $3)$, a veces (4, 5 y 6$)$, casi siempre (7, 8 y 9) y siempre (10). Esta primera adaptación se justifica porque los sujetos al ser estudiantes están acostumbrados a la escala de 0 a 10, ya que así han sido evaluados por el sistema educativo de nuestro país (México); y ii) La segunda adaptación consistió en aplicar el instrumento por medio de una computadora; esto con el fin de permitir el almacenamiento de los datos sin etapas previas de codificación, con una mayor precisión y rapidez (Figura 1).

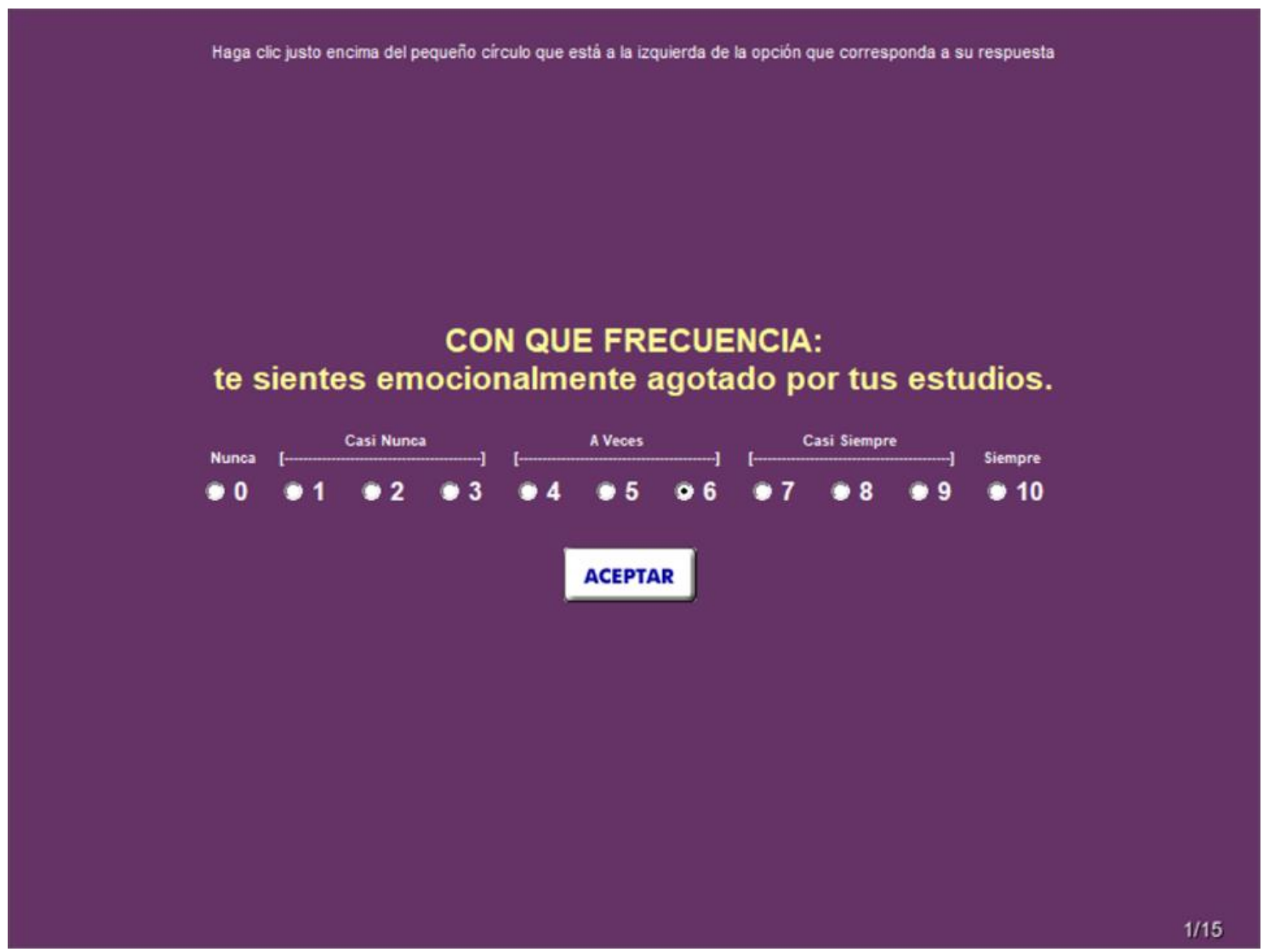

Fig. 1: Ejemplo de respuesta para los ítems del cuestionario.

\section{Diseño}

En cuanto al diseño del estudio, se utilizó un enfoque cuantitativo con un diseño descriptivo y transversal tipo encuesta. La variable independiente fue género y las variables dependientes las puntuaciones obtenidas en cada uno de los factores del Inventario de burnout para estudiantes de Maslach. La tabla 1 muestra la encuesta utilizada para la recolección de datos.

\section{Procedimiento}

Una vez conseguido el permiso de las autoridades educativas correspondientes, se invitó a participar en el estudio a los alumnos y alumnas de las Licenciaturas en Motricidad Humana y Educación Física de la Facultad 
de Ciencias de la Cultura Física de la Universidad Autónoma de Chihuahua. Los que aceptaron participar firmaron la carta de aceptación correspondiente. Luego se aplicó el instrumento por medio de una computadora personal, en una sesión de aproximadamente 30 minutos; en los laboratorios de cómputo de la Facultad de Ciencias de la Cultura Física de la Universidad Autónoma de Chihuahua. Al inicio de cada sesión se hizo una pequeña introducción sobre la importancia de la investigación y de cómo acceder al instrumento. Se les solicitó la máxima sinceridad y se les garantizó la confidencialidad de los datos que se obtuvieran. Las instrucciones de cómo responder se encontraban en las primeras pantallas; antes del primer reactivo del instrumento. Al término de la sesión se les agradeció su participación. Una vez aplicado el instrumento se procedió a recopilar los resultados por medio del módulo generador de resultados del editor de escalas versión 2.0 (Blanco et al., 2013).

\section{Análisis de datos}

Se realizaron análisis estadísticos descriptivos (medias y desviaciones estándar) para todas las variables. Posteriormente, después de verificar que los datos cumplían con los supuestos de los análisis estadísticos paramétricos, se llevó a cabo un análisis multivariante de varianza (MANOVA), seguido por los análisis de varianza univariados (ANOVAs), para examinar las diferencias entre mujeres y hombres en cuanto a las puntuaciones obtenidas en cada uno de los factores del Inventario de burnout para estudiantes de Maslach. Por otra parte, el tamaño del efecto se estimó mediante el eta cuadrado $\left(n^{2}\right)$. Todos los análisis estadísticos se realizaron con el programa SPSS versión 21.0 para Windows. El nivel de significación estadística se estableció en $p=.05$.

A continuación, se presentan las preguntas en formato, de la encuesta utilizada (Tabla1):

Tabla 1: Encuesta utilizada en este estudio para la recolección de datos

Subescala 1: Agotamiento

1. Con qué frecuencia: Te sientes emocionalmente agotado por tus estudios.

2. Con qué frecuencia: Te sientes agotado al final de un día en la universidad.

3. Con qué frecuencia: Te sientes cansado cuando despiertas en la mañana y tienes que enfrentar otro día en la universidad.

4. Con qué frecuencia: Estudiar o asistir a una clase es realmente estresante para ti.

5. Con qué frecuencia: Te sientes agotado por tus estudios.

Subescala 2: Cinismo

1. Con qué frecuencia: Te sientes sin interés en tus estudios.

2. Con qué frecuencia: Te sientes sin entusiasmo para estudiar.

3. Con qué frecuencia: Haces comentarios burlones sobre la utilidad de lo que estudias.

4. Con qué frecuencia: Dudas sobre la importancia de tus estudios.

Subescala 3: Eficacia académica

1. Con qué frecuencia: Puedes resolver eficazmente los problemas que surgen en tus estudios.

2. Con qué frecuencia: Crees que puedes hacer una contribución efectiva en las clases que asistes.

3. Con qué frecuencia: Te consideras un buen estudiante.

4. Con qué frecuencia: Te sientes entusiasmado cuando alcanzas tus metas estudiantiles.

5. Con qué frecuencia: Has aprendido cosas interesantes a lo largo de tus estudios.

6. Con qué frecuencia: Durante la clase te sientes seguro de que eres competente.

\section{RESULTADOS}

La Tabla 2 muestra los valores de las medias (Figura 2) y las desviaciones estándar de las puntuaciones de los cuatro factores estudiados sobre la percepción de barreras para la práctica de ejercicio físico, así como los resultados del MANOVA y los subsiguientes ANOVAs. Los resultados del MANOVA mostraron diferencias globales estadísticamente significativas de acuerdo a la variable género en los puntajes promedio de las subescalas de burnout académico percibido (Wilks' $\lambda=.922 ; p<.001 ; \eta^{2}=.078$ ). Posteriormente, los resultados de los ANOVAs mostraron que las mujeres reportan mayores niveles de burnout académico en las tres dimensiones estudiadas Agotamiento $(F=6.224, p<.05)$, Cinismo $(F=40.037, p<.001)$ y Eficacia $(F=$ 23.348, $p<.001)$. 
Tabla 2: Resultados MANOVA y ANOVAs para las diferencias en percepción de burnout académico de acuerdo al género. Nota. Los valores descriptivos se presentan como media (desviación estándar).

\begin{tabular}{|l|c|r|r|r|r|}
\hline & $\begin{array}{c}\text { mujeres } \\
(n=598)\end{array}$ & $\begin{array}{c}\text { hombres } \\
(n=582)\end{array}$ & \multicolumn{1}{|c|}{$F$} & \multicolumn{1}{c|}{$p$} & \multicolumn{1}{|c|}{$\eta^{2}$} \\
\hline & & & 32.235 & $<.001$ & .078 \\
\hline Agotamiento & $5.140(0.079)$ & $4.890(0.077)$ & 6.224 & $<.05$ & .010 \\
\hline Cinismo & $7.164(0.081)$ & $6.432(0.082)$ & 40.037 & $<.001$ & .033 \\
\hline Eficacia & $8.094(0.049)$ & $7.745(0.049)$ & 23.348 & $<.001$ & .021 \\
\hline
\end{tabular}

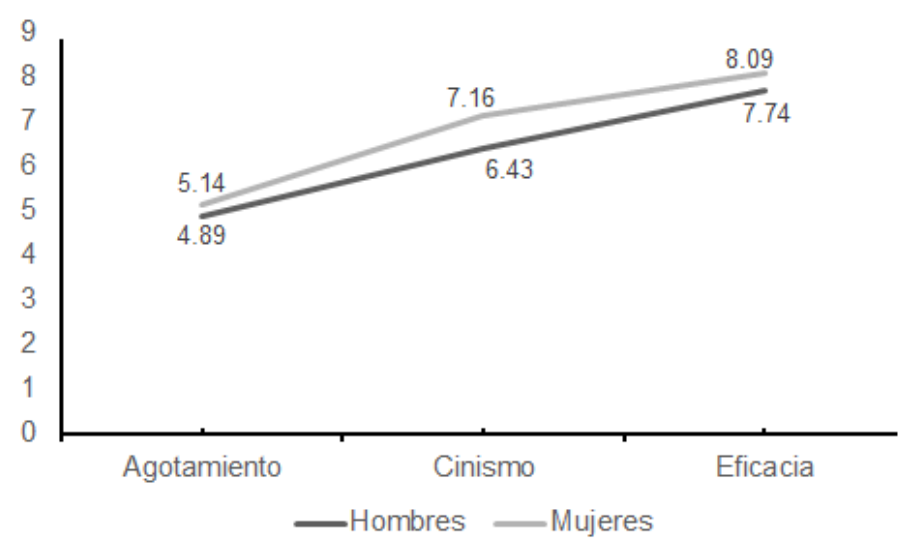

Fig. 2: Perfil de burnout académico de acuerdo al género.

\section{DISCUSIÓN}

Los resultados obtenidos revelan que en todas las áreas o factores del burnout académico, las mujeres en comparación con los hombres se perciben con mayores niveles de burnout académico, es decir, en comparación con los hombres se sienten emocionalmente más agotadas por sus estudios, con menos interés, dudan en mayor medida sobre la importancia de sus estudios y menos entusiasmadas cuando alcanzan alguna meta académica. Resultados que en general concuerda con estudios similares (Acosta et al., 2016; Go et al., 2018; LaFaver, Miyasaki, Keran, Rheaume, Gulya, Levin, et al., 2018) donde se reporta también que las mujeres tienden a manifestar niveles más altos de burnout académico que los hombres; provocando así en las mujeres una mayor presencia de sensaciones de no poder dar más de sí mismas tanto física como psíquicamente, una actitud negativa, de desvalorización, de pérdida de interés por trascender, así como mayores dudas sobre el valor del estudio y de la capacidad de lograr un buen desempeño. A diferencia de los resultados encontrados en los que los hombres fueron quienes se sintieron con más burnout que las mujeres (Jahedizadeh et al., 2015; Saville et al., 2018).

De acuerdo con Malach-Pines y Ronen (2016) sobre la base de la perspectiva biológica, incluidas las teorías evolutivas y psicoanalíticas, se puede esperar que las diferencias de género en el agotamiento sean grandes y universales. Sobre la base de la perspectiva social, incluidas las teorías de la función social y la construcción social, se puede esperar que las diferencias de género en el agotamiento varíen en los contextos culturales. En términos de su tamaño, de acuerdo con la teoría de la función social, se puede esperar que las diferencias de género en el agotamiento sean mayores que las diferencias de género. En resumen, la literatura sobre las diferencias entre hombres y mujeres en el burnout ha producido resultados inconsistentes con respecto a la fuerza y la dirección de esta relación (Purvanova y Muros, 2010).

\section{APLICACIONES PRÁCTICAS}

Disminuir el burnout de los estudiantes universitarios provocado por el hecho de que se encuentran expuestos a muchas demandas presentadas por su contexto educativo, el aprendizaje, el rendimiento académico, la sobrecarga de trabajo, la presión con el tiempo, la falta de oportunidades para la autogestión, la evaluación frecuente, la competencia entre compañeros, la irrelevancia percibida de contenidos, una enseñanza deficiente, las malas relaciones, etc., sin duda contribuye a su calidad de vida y salud, lo que justifica llevar a cabo investigaciones que incrementen nuestro conocimiento con respecto a dicha temática. Por ello, el presente estudio intenta determinar las diferencias y similitudes entre hombres y mujeres universitarios mexicanos, en cuanto a la percepción de burnout académico. Además el presente estudio será de utilidad para la aplicación en diferentes ámbitos como, por ejemplo, estudios de intervención para reducir el burnout. 


\section{CONCLUSIONES}

De los resultados mostrados, de su análisis y de su discusión, se pueden obtener las siguientes conclusiones:

Los resultados del análisis multivariante de varianza, seguido por los análisis de varianza univariados, mostraron que las mujeres en comparación con los hombres reportan mayores niveles de burnout académico en las tres dimensiones estudiadas (Agotamiento, Cinismo y Eficacia), predisponiendo así a las mujeres a una mayor percepción de sentimientos de fatiga, a una actitud de indiferencia o distante hacia los estudios y a una disminución en sus logros académicos.

Las diferencias encontradas entre hombres y mujeres con respecto a su percepción de burnout académico sugieren además, que al diseñar cualquier tipo de intervención que tenga como objetivo la disminución del mismo habrá que tomar en cuenta a la variable género. Se subraya además la importancia de realizar un mayor número de investigaciones sobre el tema en nuestro país.

Por último, cuando menos dos limitaciones están presentes en este trabajo. La primera es que los participantes son solo estudiantes universitarios mexicanos, lo que supone una amenaza para la posibilidad de generalizar estos resultados. Ampliar la muestra (agregando por ejemplo adultos jóvenes que no son estudiantes) es un área de trabajo de cara al futuro. La segunda limitación proviene del propio instrumento de medición, que se basa en el autoinforme y que por ello puede contener los sesgos que se derivan de la deseabilidad social.

\section{AGRADECIMIENTOS}

A la Secretaría de Educación Pública-Subsecretaría de Educación Superior-Dirección de Superación Académica- Programa para el Desarrollo Profesional Docente (DE-13 -6894) quién financió este estudio.

\section{REFERENCIAS}

Acosta J., R. Balado, M. E. Sardiña y A. Consuegra, Síndrome de Burnout en Estudiantes Internos de Medicina durante su Estancia por Pediatría. Hospital Pediátrico del Cerro, Panorama Cuba y Salud, 11(2), $43-48$ (2016)

Aulak D. S., B. Quinn y N. Wilson, Student Burnout, doi: 10.1038/sj.bdj.2016.156, British Dental Journal, 200(5), 219$220(2016)$

Blanco H., M. Ornelas y otros cinco autores, Editor for Creating and Applying Computerise Surveys, doi: 10.1016/j.sbspro.2013.12.105, Procedia Social and Behavioral Sciences, 106, 935-940 (2013)

Casanova J., S. Benedicto, F. Luna y C. Maldonado, Burnout, Inteligencia Emocional y Rendimiento Académico: un Estudio en Alumnado de Medicina, Revista Electrónica de Investigación Docencia Creativa, 1-6, (2016)

Castellanos, J., Gender Differences within Academic Burnout. Paper presented at the 42nd Annual Conference Proceedings (2018)

Fiorilli C., S. De Stasio y otros tres autores, School Burnout, Depressive Symptoms and Engagement: Their Combined Effect on Student Achievement, International J. Educational Research, 84, 1-12 (2017)

Freudenberger H. J., Staff Burn-out, doi: 10.1111/j.1540-4560.1974.tb00706.x, Journal of Social Issues, 30(1), 159165 (1974)

Gil-Monte, P. R., S. Unda y J. I. Sandoval, Validez Factorial del "Cuestionario para la Evaluación del Síndrome de Quemarse por el Trabajo» (CESQT) en una Muestra de Maestros Mexicanos, Salud Mental, 32(3), 205-214 (2009)

Go, H.-Y., H. K. Sung y otros tres autores, Cross-sectional Study about Stress and Health Recognition in Korean Medicine Student by Gender, doi: 10.13048/jkm.18017, Journal of Korean Medicine, 39(2), 80-91 (2018)

Gorski P. C., Fighting Racism, Battling Burnout: Causes of Activist Burnout in US Racial Justice Activists, doi: 10.1080/01419870.2018.1439981, Ethnic and Racial Studies, 1-21 (2018)

Herrera L., L. Mohamed y S. Cepero, Cansancio Emocional en Estudiantes Universitarios, Dedica. Revista de Educação e Humanidades, (9), 173-191 (2016)

Hinrichs C. P., L. E. Ortiz y C. E. Pérez, Relación entre el Bienestar Académico de Estudiantes de Kinesiología de una Universidad Tradicional de Chile y su Percepción del Ambiente Educacional, doi: 10.4067/S0718-50062016000100012, Formación Universitaria, 9(1), 109-116 (2016)

Jahedizadeh S., A. Ghanizadeh y B. Ghonsooly, The Role of Efl Learners' Demotivation, Perceptions of Classroom Activities, and Mastery Goal in Predicting their Language Achievement and Burnout, doi: 10.1186/s40862-016-0021-8, Asian-Pacific Journal of Second and Foreign Language Education, 1(1), 16 (2016)

Jahedizadeh S., A. Ghanizadeh y Z. Ghapanchi, A Cross-Contextual Analysis of Efl Students' Burnout with Respect to their Gender and Educational Level, International Journal of English and Education, 4(3), 10-22 (2015) 
Karimi Y., M. Bashirpur, M. Khabbaz y A. A. Hedayati, Comparison Between Perfectionism and Social Support Dimensions and Academic Burnout in Students, doi: 10.1016/j.sbspro.2014.12.328, Procedia-Social and Behavioral Sciences, 159, 57$63(2014)$

Kristanto T., W. S. Chen e Y.Y. Thoo, Academic Burnout and Eating Disorder among Students in Monash University Malaysia, doi: 10.1016/j.eatbeh.2016.03.029, Eating Behaviors, 22, 96-100 (2016)

LaFaver K., J. M. Miyasaki, C. y otros catorce autores, Age and Sex Differences in Burnout, Career Satisfaction, and WellBeing in Us Neurologists, doi: 10.1212/wnl.0000000000006497, Neurology (2018)

Lebares C. C., H. J. Braun y otros tres autores, Burnout and Gender in Surgical Training: A Call to Re-evaluate Coping and Dysfunction, The American Journal of Surgery (2018)

Malach-Pines A. y S. Ronen, Gender Differences in Burnout: A Literature Review, En New Directions in Organizational Psychology and Behavioral Medicine, por C. Cooper, Antoniou A. S., 107-188, Taylor \& Francis (2016)

Maslach C., Burnout: A Multidimensional Perspective, En Professional Burnout: Recent Developments in Theory and Research, por W. B. Schaufeli, Maslach C., Marek T., 19-32, Routledge (2017)

Mészaros V., S. Ádám, y otros tres autores, The Bifactor Model of Maslach Burnout Inventory-Human Services Survey (MBI-HSS) - An Alternative Measurement Model of Burnout, Streess Health, 30, 82-88 (2013)

Moneta G. B., Need for Achievement, Burnout, and Intention to Leave: Testing an Occupational Model in Educational Settings, doi: 10.1016/j.paid.2010.10.002, Personality and Individual Differences, 50, 274-278 (2011)

Morgan B., G. P. De Bruin y K. De Bruin, Operationalizing Burnout in the Maslach Burnout Inventory-Student Survey: Personal Efficacy versus Personal Inefficacy, South African Journal of Psychology, 44(2), 216-227 (2014)

Palacio J., C. Caballero y otros tres autores, Relación del Burnout y las Estrategias de Afrontamiento con el Promedio Académico en Estudiantes Universitarios, Universitas Psychologica, 11(2), 535-544 (2012)

Peralta-Ayala C.R. y T.J. Moya, Síndrome de Burnout en Estudiantes de Odontología, doi: 10.23857/dom.cien.pocaip.2017.3.1.432-442, Dominio de las Ciencias, 3(1), 432-442 (2017)

Pérez-Mármol J. M. y T. Brown, An Examination of the Structural Validity of the Maslach Burnout Inventory-Student Survey (Mbi-Ss) Using the Rasch Measurement Model, Health Professions Education (2018)

Portolés A. y J. González, Rendimiento Académico y Correspondencias con Indicadores de Salud Física y Psicológica, Sportis. Scientific Journal of School Sport, Physical Education and Psychomotricity, 1(2), 164-181 (2015)

Portolés A. y J. Hernández, Actividad Física y Niveles de Burnout en Alumnos de la ESO, Retos: Nuevas Tendencias en Educación Física, Deporte y Recreación, (29), 95-99 (2016)

Purvanova, R. K., y J. P. Muros, Gender differences in burnout: A meta-analysis, Journal of vocational behavior, 77(2), 168-185 (2010)

Richards, K. A., M. A. Hemphill y T. J. Templin, Personal and Contextual Factors Related to Teachers' Experience with Stress and Burnout, doi: 10.1080/13540602.2018.1476337, Teachers and Teaching, 24(7), 787-787 (2018)

Saville, B. K., A. Bureau, C. Eckenrode y M. Maley, Passion and Burnout in College Students. College Student Journal, 52(1), 105-117 (2018)

Seibert, G. S., K. N. Bauer, R. W. May y F. D. Fincham, Emotion Regulation and Academic Underperformance: The Role of School Burnout, doi: 10.1016/j.lindif.2017.10.001, Learning and Individual Differences, 60, 1-9 (2017)

Serrano F. T., J. Salguero-Sánchez y otros cuatro autores, Síndrome de Burnout en Estudiantes de 6 Facultades de Medicina de Colombia, 2016-1: Estudio Multicéntrico, Ciencia e Investigación Medico Estudiantil Latinoamericana, 21(2), 29-34 (2016)

Shaufeli, W. B., I., Martínez y otros tres autores, Burnout and Engagement in University Students: A Cross-national Study, Journal of Cross-Cultural Psychology, 33(5), 464-481 (2002)

Shaufeli W. B., M. Leiter y C. Maslach, Burnout: 35 Years of Research and Practice, doi: 0.1108/13620430910966406, Career Development International, 14(3), 204-220 (2009)

Tement S., A. Pahor y N. Jaušovec, EEG Alpha Frequency Correlates of Burnout and Depression: The Role of Gender, Biological psychology, 114, 1-12 (2016)

Vizoso C. M. y O. Arias, Estresores Académicos Percibidos por Estudiantes Universitarios y su Relación con el Burnout y el Rendimiento Académicos, doi: 10.1016/j.anpsic.2016.07.006, Anuario de Psicología, 46(2), 90-97 (2016) 